\title{
DIEP, DEEP TROUBLE
}

\author{
$P$ Kouwenhoven ${ }^{1}$
}

Delft, The Netherlands

The global village evolving through the ever-growing Internet community allows for all kinds of confrontations between people with differing views and ideas. One of these battlegrounds is the rec.games.chess.computer (r.g.c.c.) newsgroup, where discussions on computer chess are going on, algorithms are born and claims are made. Recently, one of the contributors, Vincent Diepeveen, announced the availability of his new Homepage (http://www.students.cs.ruu.nl/ vdiepeve/), with information on his chess program DIEP. He invited people to come and take a look, proclaiming some surprises. On Vincent's homepage the following claim was made "DiEP is the best chess program in the world, and given a sufficient amount of time, it can beat any other program." This claim started a series of events, eventually forcing Vincent to change the contents of his WWW pages, but still (May 13, 1997) the following statement can be found: "In analyzing positions DIEP is by far the strongest chess program in the world." Many chess programmers took offense in this claim, and urged Vincent to prove this bold statement. Eventually, he produced a series of positions in which DIEP seemed to perform quite well. One of the more regular contributors of r.g.c.c., using the pseudonym of "Komputer Korner" suggested that a match should be played in which DIEP would be allowed to try to prove against other chess programs that it is indeed the "best" program in the world. This match should be played on a common PC platform (a $66 \mathrm{MHz} 80486$ computer) allowing volunteers to participate in parallel. So many games would run on at the same time. Eight hours of computing time per move was considered to be "sufficient amount of time" and would allow operators to sleep. The Komputer Korner Korrespondence Kup was born.

New questions and discussions developed on the following issues:

- Which computers should participate?

- Why using only Intel-platform (80486 and Pentium) programs?

- Which playing rate should be used?

- How to deal with the differences in speed for the different computers?

- What should be the size of the hash tables?

Finally when the dust settled, the following rules were established ("Adapted rules for the Korrespondence Kup of 2 February 1997"):

1. Every program plays at a rate of 8 hours per move, unless the computer is faster than a $486 / 66$, in which case a handicap is used. So, the faster the machine, the less time is allotted.

2. A program cannot lose on time. Every operator is honest and acts in the name of science. Do not feel uncomfortable when a move is late, the opponent can be busy with other games, off for the weekend, or having holidays.

3. The participants are: DIEP 1.57.17, REBEL 8, CRAFTY 11.15, MACCHESS 3, and CM5000 1.0.2.

4. All five participants are playing in parallel, so every program plays four games at the same time, possibly by different operators.

5. The winner is the program with the most points. After all results are established, the winner receives the "Komputer Korner Korrespondence Gold Kup". The winner of a game communicates the result, in case of a draw, the white player does.

6. "Komputer Korner" is not an operator, but acts as the Tournament Director. For arbitration see rule 8.

7. "Komputer Korner" will publish a list of participants and programs. Played moves can be posted in r.g.c.c and checked by others.

8. Games not finished by 1 December, 1997 are adjudicated. The tournament starts on Saturday, 8 February, 1997. Arbiters are Bruce Moreland, Tom Kerrigan and John Stanback (if they are so kind to make themselves available). Hopefully arbitration is not needed. If your program is winning, do not manipulate the playing rate. It is a scientific test. If manipulation is detected, the result is declared void.

1 Resource Analysis, Zuiderstraat 110, 2611 SJ Delft, The Netherlands. Email: peter kouwenhoven@resource.nl. 
9 There is a maximum of four operators per program This should speed up things, because it is not necessary to use $4 \times 8$ hours per move

10 For every program, only the default standard settıngs are used There is a maximum size for the hash tables

11 "Spectators" should be able to reproduce the results

12 In case an operator is retreating, he/she will be replaced

13 Every position will be evaluated with the "permanent brain" turned off (because of the different time handicaps) Every program will play if the position was just reached Opening books are allowed The used amounts of RAM, MHz and internal caches must be reported

14 Every settıng must be published

15 The basic machine is a 486/66 Faster machınes get a tıme handicap, established by the "arbiters committee"

16 When a move is received, this should be confirmed by the opponent

17 Not only the move made, but the whole game (in PGN format) should be communicated

18 Once the tournament is started, changes in software or hardware are not allowed Every move played must be stored in a game history

19 Operators neither offer nor accept draws This has to be performed by the programs Operators may not interfere The operator is not allowed to fix the opening

The following time handicaps were establıshed

CM5000.

$\begin{array}{ll}\text { Pentium } 75 & -4 \text { hours } 10 \text { minutes } \\ \text { NEXGEN 586/90Mhz } & -3 \text { hours } 20 \text { minutes } \\ \text { P PRo/200 } & -1 \text { hour }\end{array}$

CRAFTY:

P Pro/200 Mhz - 58 minutes

Sun ULtra $143 \mathrm{Mhz} \quad-2$ hours 15 minutes

$80486100 \mathrm{Mhz} \quad-5$ hours 20 minutes

DIEP

$8048666 \mathrm{Mhz} \quad-8$ hours

The sizes of the hash tables are configured as follows

$\begin{array}{ll}\text { CM5000 } & -419 \mathrm{Mb} \\ \text { CRAFTY } & -6 \mathrm{Mb} \text { general hash and } 1 \mathrm{Mb} \text { Pawn hash } \\ \text { DIEP } & -57 \mathrm{Mb} \\ \text { MACCHESS } & -4 \mathrm{Mb} \\ \text { REBEL } & -6 \mathrm{Mb}\end{array}$

The tournament consisted of ten games The distribution of colours is given in Table 1 together with the platforms used in the particular game Considering the fact that in principle any program could participate it stikes the author that four of the five programs are Dutch CM5000 by Johan de Konıng, REBEL by Ed Schroder, DIEP by Vincent Diepeveen, and MACCHESS by Wim van Beusekom Only CRAFTY by Robert Hyatt is American In my opinion, this points to a kind of Dutch supremacy in computer chess

\begin{tabular}{|l|l|l|}
\hline CM5000 - REBEL 8 & Pentium 75 & P-200 \\
\hline CRAFTY - MACCHESS 3 & P Pro/200 & PowerMac 6100/66 \\
\hline CM5000 - DIEP & P Pro/200 & $80486 / 66$ \\
\hline REBEL 8 - CRAFTY & P133 & P Pro/200 \\
\hline MACCHESS 3 - CM5000 & PowerMac 7200/90 & P Pro/200 \\
\hline REBEL 8- DIEP & $80486 / 66$ & $80486 / 66$ \\
\hline CRAFTY - CM5000 & Sun Ultra/143Mhz & NexGEN 586/90Mhz \\
\hline DIEP - MACCHESS 3 & $80486 / 66$ & Power Mac 6100/66 \\
\hline MACCHESS 3 - REBEL 8 & PowerMac 7200/200 & P-133 \\
\hline DIEP - CRAFTY & $80486 / 66$ & $80486 / 100$ \\
\hline
\end{tabular}

Table 1: The playing scheme

\author{
MACCheSS: \\ PowerMac 6100/66 - 4 hours 10 minutes \\ PowerMac 7200/90 - 2 hours 55 minutes \\ REBEL: \\ $\begin{array}{ll}\text { P133 } & -8 \text { hours } \\ & -2 \text { hours } 25 \text { minutes }\end{array}$ \\ Pentium $200 \quad-1$ hour 30 minutes
}


The final standings are given in Table 2

\begin{tabular}{|l|c|c|c|c|c|c|}
\hline & CM5000 & CRAFTY & REBEL 8 & DIEP & MACCHESS 3 & Total \\
\hline CM5000 102 & $\mathrm{X}$ & 0 & 05 & 1 & 1 & 25 \\
\hline CRAFTY 11 15 & 1 & $\mathrm{X}$ & 05 & 0 & 1 & 25 \\
\hline REBEL 8 & 05 & 05 & $\mathrm{X}$ & 1 & 05 & 25 \\
\hline DIEP 1 57 17 & 0 & 1 & 0 & $\mathrm{X}$ & 1 & 2 \\
\hline MACCHESS 3 & 0 & 0 & 05 & 0 & $\mathrm{X}$ & 05 \\
\hline
\end{tabular}

Table 2: The final standings

\section{REBEL 8 - CRAFTY}

1 e4 e5 2 Nf3 Nc6 3 d4 d6 4 Bb5 Bd7 5 Nc3 Nf6 6 0-0 Be7 7 Bxc6 Bxc6 8 Rel exd4 9 Nxd4 Bd7 10 Bg5 0-0 11 Qd2 h6 12 Bf4 Re8 13 Rad1 Nh5 14 Be3 Bf6 15 Qe2 Bxd4 16 Rdxd4 Nf6 17 f3 Bc6 18 Red1 Qe7 19 a3 Qe5 20 b4 a6 21 R4d3 Qh5 22 Qf2 Rac8 23 Ne2 Bb5 24 Ng3 Qg6 25 Rd4 Ba4 26 Ne2 Nd7 27 Nc3 Bc6 28 Nd5 Bxd5 29 Rxd5 Ne5 30 Bf4 Nc4 31 Qa7 Qf6 32 Bc1 Rb8 33 Rd5d3 Rec8 34 f4 c5 35 bxc5 dxc5 36 e5 Qc6 37 Rd7 Na5 38 R7d6 Qe4 39 R6d3 Qg6 40 Rd6 Qxc2 41 R6d3 Nb3 42 Be3 Qe2 43 e6 fxe6 44 Qb6 e5 45 Kh1 Nd4 46 fxe5 Rf8 47 Qb1 c4 48 Rxd4 Qxe3 49 Rxc4 Qxe5 50 Re4 Qc3 51 a4 Rf2 52 Rg1 Rb2 53 Qd1 Qc6 54 Qg4 Re8 55 Rxe8+ Qxe8 56 h3 Kh2 57 Rd1 Qg6 58 Qxg6 Kxg6 59 Kh2 Rb4 60 a5 Rb5 61 a5 Rb5 62 axb6 Rxb6 63 Ra5 Kf7 64 Kg3 Rf6 65 Re5 g6 66 h4 Rf5 67 Re3 Rf1 68 Re5 Rd1 69 Ra5 Rd6 70 Kf4 Rf6+ 71 Ke4 h5 72 Rc5 Ke7 73 Rc7+ Kd6 74 Rc8 Rf2 75 Ra8 Ra2 76 Kf3 Ke5 77 Re8+ Kf5 78 Rf8+ Ke6 79 Re8+ Kf6 80 Rc8 Ra5 81 Rc6+ Kf5 82 Kf2 Ra2+ 83 Kg3 Ra3+ 84 Kh2 a5 85 Rc5+ Kf6 86 Rc6+ Kg7 87 Rc7+ Kh6 88 Ra7 a4 89 Kgl g5 90 Ra6+ Kg7 91 hxg5 Ra1+ 92 Kh2 a3 1⁄2-1/2

\section{CM5000 - DIEP}

1 d4 d5 2 Nf3 Nf6 3 c4 c6 4 Nc3 a6 5 e3 (CM5000 out of book) b5 6 cxd5 cxd5 7 Bd3 e6 8 e4 dxe4 (DiEP out of book) 9 Nxe4 Bb4+ 10 Bd2 Bxd2+ 11 Qxd2 Bb7 12 Nxf6+ Qxf6 13 Rcl Nd7 14 Be2 Bd5 15 0-0 0-0 16 b3 Rfc8 17 a4 Rcb8 18 Rc3 b4 19 Re3 a5 20 Bb5 Nb6 21 Ne5 Rc8 22 Rh3 h6 23 Ng4 Qg6 24 Rg3 Kh8 25 Ne5 Qf6 26 Re1 Rc7 27 Rh3 Qe7 28 Rxh6+ Kg8 29 Re3 gxh6 30 Rg3+ Kh7 31 Nxf7 Qxf7 32 Bd3+ Qf5 33 Bxf5+ exf5 34 Qf4 Rg7 35 Rxg7+ Kxg7 36 Qc7+ Bf7 37 Qxb6 Rc8 38 h4 Rc1+ 39 Kh2 Bxb3 40 Qxa5 Bf7 41 Qxb4 Rc6 42 a5 Ra6 43 Qb5 1-0

\section{REBEL 8 - DIEP}

1 e4 e6 2 d4 d5 3 Nc3 Nf6 4 Bg5 Be7 5 e5 Nfd7 6 Bxe7 Qxe7 7 f4 0-0 8 Nf3 c5 9 Qd2 Nc6 10 0-0-0 Nb6 11 dxc5 Qxc5 $12 \mathrm{~Kb} 1 \mathrm{Bd} 713 \mathrm{Bd} 3 \mathrm{Rfc} 814 \mathrm{Nb} 5$ f6(both out of book) 15 exf6 gxf6 16 c3 Qe7 17 Rhel e5 18 fxe5 fxe5 19 c4 dxc4 20 Be4 Qf6 21 Nd6 Rc7 22 Rf1 c3 23 bxc3 h6 24 Ng1 Qh4 25 g3 Qg4 26 Rf7 Nc4 27 Bh7+ Kh8 28 Nxc4 Nd8 29 Nxe5 Nxf7 30 Nxg4 Bxg4 31 Qf4 Bxd1 1-0

\section{CRAFTY - CM5000}

Nf3 d5 2 d4 Nf6 3 c4 dxc4 4 e3 e6 5 Bxc4 c5 6 0-0 a6 7 a4 Nc6 8 Qe2 Qc7 9 Nc3 Bd6 10 Rd1 0-0 11 h3 Re8 12 dxc5 Bxc5 13 e4 Nd7 14 Ba2 b6 15 e5 Ndxe5 16 Bf4 Nxf3+ 17 Qxf3 (CRAFTY out of book) e5 18 Nd5 Qb7 19 Nf6+ gxf6 20 Bh6 Bf8 21 Bd5 Bd7 22 Ra3 Bxh6 23 Qxf6 Re6 24 Rg3+ Kf8 25 Bxe6 Bxe6 26 Qxh6+ Ke7 27 Qh4+ Kf8 28 Rd6 Qc8 29 Rc3 Bd7 30 Qxh7 Ke7 31 Qh6 e4 32 Qf6+ Kf8 33 Qh4 Qd8 34 Qh6+ Ke8 35 Rcxc6 Bxc6 36 Qh8+ Ke7 37 Qe5+Kf8 1-0

\section{CM5000 - REBEL 8}

$1 \mathrm{~d} 4 \mathrm{~d} 52 \mathrm{Nf} 3 \mathrm{c} 5$ (CM5000 out of book) 3 dxc5 e6 4 b4 a5 5 c3 axb4 (REBEL8 out of book) 6 cxb4 b6 7 Qc2 Nd7 8 c6 Bxb4+ 9 Nbd2 Nc5 10 Rb1 Ba5 11 e3 Ne7 12 Bb5 Qc7 13 Bb2 f6 14 0-0 e5 15 Rbd1 Bf5 16 Qc1 Bd3 17 BxBd3 Nxd3 18 Qc2 Nxb2 19 QxNb2 Qxc6 20 Rc1 Qd6 21 Rfdl 0-0 22 Nc4 Qe6 23 Nxa5 Rxa5 24 Qb3 Rfa8 25 Rc2 Rc5 26 Ne1 Kf7 27 Nd3 Rxc2 28 Qxc2 Qg4 29 Rd2 Rc8 30 Qb1 Qe4 31 Qb3 Qc4 32 Qb1 Rc6 33 Rb2 Nc8 34 Qd1 g6 35 Nb4 Rc5 36 Nd3 Rc7 37 h3 Qe4 38 Rb5 Rc3 39 Ne1 Ne7 40 Qd2 Rc6 41 Rb4 Qf5 42 Qb2 Nc8 43 Qd2 Qe6 44 Rb1 Nd6 45 Rd1 Rc5 46 Nd3 Ra5 47 Nb4 Nc4 48 Qc3 Kg7 49 Qb3 Qf7 50 a4 h6 51 Rd3 Qe8 52 Rxd5 Qxa4 53 Qxa4 Rxa4 54 Rd7+ Kf8 55 Nd5 Ra1+ 56 Kh2 Rd1 57 e4 f5 58 exf5 gxf5 59 Nxb6 Rxd7 60 Nxd7+ Ke7 61 Nc5 Kd6 62 Nd3 Kd5 63 Kg3 Kd4 64 Ne1 Nb2 65 Nf3+ Kd5 66 Nh4 Ke6 67 Kf3 Kf6 68 g4 fxg4 69 hxg4 Kg5 70 Kg3 Nc4 71 Nf3 1/2-1/2 (draw by agreement')

\section{DiEP - CRAFTY}

1 e4 e5 2 Nf3 Nc6 3 Bb5 a6 4 Ba4 Nf6 5 0-0 Nxe4 6 d4 b5 7 Bb3 d5 8 dxe5 Be6 9 Qe2 Be7 10 Rd1 0-0 11 c4 bxc4 12 Bc4 Bc5 13 Be3 Bxe3 14 Qxe3 Qb8 15 Bb3 Na5 16 Nbd2 Qa7 17 Qxa7 Rxa7 18 Nxe4 Nxb3 19 axb3 dxe4 20 Nd4 Rb7 21 Rxa6 c5 22 Nxe6 fxe6 23 Rxe6 Rxb3 24 Rd2 Rfb8 25, Rc6 Rxb2 26 Rxb2 Rxb2 27 h4 Rb1+ 28 Kh2 Rb5 29 Kg3 Kf7 30 Kf4 Rb2 31 Ke3 Rc2 32 h5 Rc4 33 h6 gxh6 34 Rxh6 Rd4 35 g3 Kg7 36 Rh4 Rb4 37 Rf4 h6 38 e6 Rb6 39 e7 Rb8 40 Rf8 1-0 


\title{
DIEP - MACChESS 3
}

1 d4 d5 2 Nf3 Nf6 3 c4 e6 4 e3 c5 5 Nc3 Ne4 (MACCHESS 3 out of book) 6 Bd3 (DIEP out of book) Nxc3 7 bxc3 dxc3 8 Bxc4 cxd4 9 exd4 Qc7 10 Qd3 Nc6 11 0-0 Bd6 12 Rfe1 Bd7 13 d5 Ne5 14 Nxe5 Bxe5 15 Ba3 f6 16 dxe5 Bxh2+ 17 Kf1 Ba4 18 Rad1 Be5 19 Bb5+ Bxb5 20 Qxb5+ Qc6 21 Qb4 Qa6+ 22 Re2 Qxe6 23 Rd6 Qxd6 24 Qxd6 Kf7 25 Rxe5 Rae8 6 Qd7+ Kg6 27 Rxe8 Rxe8 28 Qxe8+ Kf5 29 Bc1 Kg4 30 f3+ Kh4 31 Qa4+ Kg3 32 Qf4+ 1-0

\section{CRAFTY - MaCChESS 3}

1 e4 Nf6 2 Nc3 d5 3 e5 d4 4 exf6 dxc3 5 fxg7 cxd2+ 6 Qxd2 Qxd2+ 7 Bxd2 Bxg7 8 0-0-0 Nc6 9 Bb5 Bd7 10 Ne2 a6 11 Ba4 Rg8 12 Nf4 Bd4 13 Nd5 (CRAFTY out of book) Rc8 14 Bf4 b5 15 Bb3 Kd8 16 Bg3 Bg7 17 Nf4 Rf8 18 Rhe1 Ke8 19 Nd5 e5 20 f4 Bg4 21 Rd3 f6 22 Bf2 Na5 23 Rg3 h5 24 h3 Nxb3+ 25 axb3 c6 26 Nb6 Bh6 27 Be3 Bxh3 28 Rxh3 Rb8 29 g3 Rh8 30 Kb1 Bg7 31 fxe5 fxe5 32 Reh1 Kf7 33 Rxh5 Rxh5 34 Rxh5 Rd8 35 Kc1 e4 36 b4 Rd6 37 Bf4 Re6 38 c3 Kg6 39 Rh4 Re8 40 Kd2 Bf6 41 Rh6+ Kg7 42 Ke3 Re7 43 Nc8 Re8 44 Nd6 Re6 45 Nf5+ Kf7 46 g4 Be5 47 Rxe6 Kxe6 48 Nd4+ Bxd4+ 49 cxd4 Kd5 50 g5 Ke6 51 Kxe4 Kf7 52 Kf5 Kg7 53 g6 Kg8 54 Kf6 Kf8 55 Bh6+ Ke8 56 g7 $\mathrm{Kd} 757 \mathrm{~g} 8=\mathrm{Q}$ 1-0

\section{MACCHESS 3 - CM5000}

1 e4 c5 2 Nc3 Nc6 3 g3 g6 4 Bg2 Bg7 5 d3 e6 6 f4 Nge7 7 Nf3 d5 8 0-0 b6 9 Bd2 Bb7 10 Rb1 Nd4 11 Ne5 f6 (CM5000 out of book) 12 Nf3 (MACCHESS 3 out of book) Nxf3+ 13 Bxf3 d4 14 Ne2 f5 15 Ncl 0-0 16 Qe2 Qd7 17 Nb3 fxe4 18 Bxe4 Bxe4 19 Qxe4 Nd5 20 Rf2 a5 21 Rel Rae8 22 Rfe2 Qb5 23 Rbl a4 24 Nc1 Qd7 25 Qg2 e5 26 fxe5 Bxe5 27 Kh1 Rf5 28 Re1 Ref8 29 Kg1 Ne3 30 Bxe3 dxe3 31 Ne2 Rf2 32 Qh1 R8f3 33 d4 cxd4 34 Qxf3 Rxf3 35 Rf1 Qg4 36 Rxf3 Qxf3 37 Nf4 Qf2+ 38 Kh1 Bxf4 39 gxf4 Qxc2 0-1

\section{MACCHESS 3 - REBEL 8}

1 d4 d5 2 c4 c6 3 cxd5 cxd5 4 Nf3 Nf6 5 Nc3 Nc6 6 Bf4 Bf5 7 e3 e6 8 Bd3 Bxd3 9 Qxd3 Bd6 10 Bg5 (MAcChess 3 out of book) 0-0 11 0-0 Rc8 12 Rfc1 a6 13 a3 h6 14 Bxf6 Qxf6 15 Rc2 Na5 16 Racl Qg6 17 Qxg6 fxg6 18 Nd2 b5 19 Na2 Rxc2 20 Rxc2 Nc4 21 Nxc4 bxc4 22 Nc3 Bb8 23 h4 Kf7 24 g4 Bc7 25 g5 Ke7 26 Kg2 h5 27 Ne2 Rb8 28 f4 Rb3 29 Kf3 Ba5 30 Nc1 Rb7 31 Kf2 Kd6 32 Kf1 Rb8 33 Kf2 Kd7 34 Ke2 Rb7 35 Kf2 Ke7 NO DRAW1 The Tournament Director decided that the game should go on, after a discussion in the newsgroup' The following moves are $36 \mathrm{Ke} 2 \mathrm{Bb} 637 \mathrm{Kf} 3 \mathrm{Kd} 6$ 38 Kg3 Bd8 39 Kf2 Kd7 40 Ne2 Kc6 41 Nc1 Kb5 42 Ke2 Ka4 43 Kd1 Ba5 44 Re2 Bb6 45 Kc2 Kb5 46 Na2 Bc7 47 Nc3+ Kc6 48 Na4 Bd6 49 Nc3 Rb8 50 Kd1 Bc7 51 Rc2 Rb6 52 Ke1 Rb3 53 Ke2 Bd8 54 Na2 The operators are again requestıng a draw, as there was no pawn advance? $1 / 2-1 / 2$

There are already plans to start another match, with more participants and higher speed platforms

\section{BOARD GAMES IN ACADEMIA: PLATFORM OF INTERNATIONAL BOARD GAMES RESEARCH}

\author{
$A$ de Voogt 1
}

Baarn, The Netherlands

On Sunday evening April 6, 1997 the third Colloquium on Board Games in Academia started off in fine style in the $17^{\text {th }}$ Century Snouck Hurgronje house at the Rapenburg in Leiden, the Netherlands Its friendly atmosphere was enhanced by cello and violın music Of course, it was a reunion of the participants of the second International Colloquium (1995), although many new faces joined the gathering The evenıng was the start of an anımated conversation contınuing untıl Thursday April 10, only interrupted by interesting lectures and little sleep

On Monday, April 17, new researchers in the field of Draughts, Rythmomachia, Inca, Maya and Egyptian board games presented their research, mainly from a linguistics point of view Many new books were added to the book display and the exchange of information was more than the organisation had hoped for

On Tuesday, Aprıl 8, Dr Eagle and Dr Finkel presented their interestıng findings, followed by Dr Matsubara from Japan and International Grandmaster Averbakh from Russia It appeared a truly international and diversıfied lecture day The theme of the lectures mostly involved classification All lectures were concluded by a lively discussion about classification for general purposes It was directed by Richard Balham

1 Da Costalaan 1, 3743 HT Baarn, The Netherlands 